\title{
Cuidado de enfermagem na perspectiva do sujeito do inconsciente e sua contribuição ao Projeto Terapêutico Singular
}

Laís Angelina Pintor ${ }^{1}$

Vanessa Pellegrino Toledo ${ }^{1}$

Ana Paula Rigon Francischetti Garcia ${ }^{1}$

Objetivo: construir uma proposta de cuidado de enfermagem a partir da leitura do sujeito do inconsciente, por meio do método da Construção de Caso Clínico em Saúde Mental, apontando sua contribuição para o Projeto Terapêutico Singular. Método: realizou-se a construção de caso clínico de um paciente frequentador de um Centro de Atenção Psicossocial da rede municipal de saúde de Campinas. Resultados: foram ordenados, por cotejamento, os principais significantes do discurso do sujeito, da família e da instituição. Conclusão: o cuidado de enfermagem ao paciente psicótico, fundamentado na singularidade, possibilita construir novos efeitos terapêuticos, pode favorecer que o paciente retome sua posição de sujeito e traz a contribuição do enfermeiro ao Projeto Terapêutico Singular.

Descritores: Saúde Mental; Enfermagem Psiquiátrica; Psicanálise; Individualidade. 


\title{
Nursing care based on the perspective of the subject of the unconscious and its contribution to the Singular Therapeutic Project
}

\begin{abstract}
Objective: to build a proposal of nursing care from the perspective of the subject of the unconscious through the method of the Construction of Clinical Case in Mental Health and pointing out its contribution to the elaboration of the Singular Therapeutic Project. Method: the clinical case of a patient attending a CAPS of the municipal health services of Campinas was carried out. Results: the main signifiers of the subject, family and institution discourse were ordered by collation. Conclusion: Nursing care for the psychotic patient based on the singularity allows the construction of new therapeutic effects, which may favor the restoration of the patient in his position of subject and bring the nurse's contribution for the Singular Therapeutic Project.
\end{abstract}

Descriptors: Mental Health; Psychiatric Nursing; Psychoanalysis; Individuality.

\section{Cuidado de enfermería pautado en la perspectiva del sujeto del inconsciente y su contribución al Proyecto Terapéutico Singular}

Objetivo: construir propuesta de cuidado de enfermería a partir de la lectura del sujeto del inconsciente por el método de la Construcción de Caso Clínico en Salud Mental y señalar su contribución en elaborar el Proyecto Terapéutico Singular. Método: se realizó la construcción de caso clínico de un paciente frecuentador de un CAPS de la red municipal de salud de Campinas. Resultados: fueron ordenados, por el cotejo, los principales significantes del discurso del sujeto, de la familia y la institución. Conclusión: El cuidado de enfermería al paciente psicótico fundamentado en la singularidad posibilita la construcción de nuevos efectos terapéuticos, pudiendo favorecer la reanudación del paciente en su posición de sujeto y aporta la contribución del enfermero al Proyecto Terapéutico Singular.

Descriptores: Salud Mental; Enfermería Psiquiátrica; Psicoanálisis; Individualidad.

\section{Introdução}

Após a reforma psiquiátrica, novos modelos de atenção à saúde mental foram adotados. O enfermeiro passou a ocupar o lugar de agente terapêutico, realizando ações voltadas não mais à tutela do paciente, mas a compreender a relação entre sofrimento psíquico, político e social(1).

Dessa forma, ao adotar-se novo olhar sobre o paciente, considerando sua individualidade, é possível que rotinas, normas e padronizações deixem de ser rígidas e se voltem para o que é possível de ser articulado a partir da demanda do sujeito, tornando-o foco e dirigente da cena do cuidado(2). Nesse contexto, pressupõe-se que a atenção psicossocial se utiliza de um processo social complexo que se transforma permanentemente, pelo surgimento de novos elementos, favorecendo a investigação de novos caminhos e meios de cuidados $^{(3)}$.

Uma forma de atingir tal cuidado é por meio da elaboração e implementação do Projeto Terapêutico Singular (PTS), que é entendido como uma estratégia de cuidado integral, organizada por ações desenvolvidas por uma equipe multidisciplinar, definida a partir da singularização das necessidades do indivíduo, considerando seu contexto social, econômico, familiar, biológico, cultural e psicológico, além de visar ao resgate de sua cidadania(4).

Ao adotar-se o discurso do cuidado como dado, pelas relações únicas estabelecidas entre enfermeiro 
e paciente ${ }^{(5)}$, possibilita-se um cuidado e uma clínica de enfermagem articulada com as necessidades dos sujeitos, considerando que sua diferença e demanda dizem respeito à manifestação inconsciente ${ }^{(6)}$. Neste estudo, portanto, o singular do sujeito é adotado sob a perspectiva da determinação por meio da manifestação do inconsciente ${ }^{(7)}$.

O inconsciente é objeto de estudo da psicanálise e caracteriza-se a partir da estruturação de uma linguagem, possuindo significantes e significados sobre os quais o discurso do Eu não possui qualquer controle. A subjetividade do sujeito se expressa em como ele articula sua cadeia de significantes, fazendo a experiência subjetiva ser sempre singular e regida pelas leis da linguagem que guiarão o funcionamento do inconsciente: cada sujeito atribui determinado sentido a cada significante(8).

Dessa forma, o acesso ao inconsciente pode ocorrer por meio da linguagem, nos lapsos, nos atos falhos, nos chistes e no sintoma. O propósito da psicanálise, nesse sentido, é explicitar essa lógica do inconsciente, na temporalidade da retroação, na qual o sujeito se apresenta como efeito singular da linguagem ${ }^{(6,9)}$.

Para a clínica da enfermagem, pode-se utilizar uma escuta na abordagem psicanalítica, que tem como princípio criar um espaço para que a palavra seja dita, circule e compareça no discurso, valendose como meio de emergência de um desejo inédito e único ${ }^{(6)}$. O sintoma, nessa perspectiva, é a formação do inconsciente, que precisa ser lida como discurso do sujeito, e entendida como resposta a ser cifrada na dinâmica da transferência, pelo intermédio do Sujeito Suposto Saber ${ }^{(10)}$, no caso, o enfermeiro.

Considerando, portanto, todos os aspectos descritos até agora, coloca-se como questão: como estabelecer um cuidado de enfermagem considerando as particularidades do sujeito e sua contribuição para criar e implementar o PTS?

Este trabalho justifica-se pela possibilidade de construir um estilo de cuidar em enfermagem que implique abordar o paciente a partir da leitura do sujeito do inconsciente, de forma a incorporar estratégias e modalidades terapêuticas favorecedoras da retomada desse sujeito em sua posição reflexiva, produtiva e de portador de um desejo, além de situar o cuidado de enfermagem, na construção do PTS, em uma dimensão multidisciplinar.

Por fim, foi estabelecido como objetivo deste estudo construir uma proposta de cuidado de enfermagem a partir da leitura do sujeito do inconsciente, por meio do método da Construção de Caso Clínico, apontando sua contribuição para a elaboração do PTS.

\section{Materiais e Método}

Trata-se de uma pesquisa qualitativa desenvolvida a partir da construção de um caso clínico em saúde mental (CCC-SM) já encerrado e que toma como referencial teórico a psicanálise, a partir do reconhecimento do sujeito dividido e da determinação do inconsciente $e^{(7,10)}$.

Para tanto, foram realizadas 11 consultas de enfermagem com um paciente frequentador de um Centro de Atenção Psicossocial (Caps) da rede municipal de saúde de Campinas, no período de 2014 a 2015 .

O método adotado, de CCC-SM, é definido como uma dialética entre as partes envolvidas na clínica do caso, na qual o paciente é colocado em trabalho, sendo importante registrar seus movimentos e recolher tudo que lhe é dito, para que o enfermeiro esteja pronto a escutar a sua palavra, quando esta vier ${ }^{(11-12)}$.

Assim, a construção do caso é um trabalho que tende a trazer à luz a relação do sujeito com o Outro, visando construir o diagnóstico do discurso e não o do sujeito. A construção serve para operar o deslocamento do sujeito dentro do discurso. O material inconsciente aparecerá sob a forma de significantes e o enfermeiro os articulará, compondo um conjunto marcado pela lógica do inconsciente daquele sujeito(13).

O significante é aquilo que não necessariamente está ligado à realidade do mundo, uma vez que a representação da coisa em si possui um objeto inconsciente que faz com que se encontre um sentido, e seja, assim, significante para uma determinada situação(14). O significado, por sua vez, é um efeito do significante e é criado por sua combinação, fazendo o sentido surgir através da substituição de um significante por outro, por meio da ação inconsciente ${ }^{(15)}$. Dessa forma, a escuta analítica, contextualizada neste trabalho nas consultas de enfermagem, está ligada ao desfile dos significantes que o paciente produz em seu discurso, definido pelo falar, às atitudes e aos comportamentos ${ }^{(8)}$.

Ao adotar o método de CCC-SM, responde-se ao cotejamento, no qual os resultados são ordenados a partir dos discursos do sujeito, da família e da instituição(16).

A análise dos dados deste estudo foi realizada em duas etapas. A primeira caracteriza-se pela leitura dos registros dos atendimentos e do material acadêmico produzido (relatórios com o conteúdo de cada consulta de enfermagem e associação teórico-prática equivalente), seguida pela segunda etapa, de releitura das consultas, respeitando-se a livre associação, possibilitando o surgimento dos significantes ${ }^{(14)}$.

A livre associação é caracterizada pela livre ocorrência de pensamentos quando o sujeito (nesse 
caso, o pesquisador) versa sobre um tema(17) (nesse caso, a leitura das consultas de enfermagem), assim, o enfermeiro opera pela transferência da identificação dos significantes contidos no discurso do paciente, utilizando o raciocínio clínico entre teoria e prática para estabelecer diagnóstico estrutural e problemas de enfermagem que alicercem as intervenções ${ }^{(9)}$.

Vale destacar que para a CCC-SM que toma o sujeito pela perspectiva da psicanálise, deve-se considerar a recomendação de não se tomar um caso em andamento como objeto de pesquisa, devido ao risco de o enfermeiro ou pesquisador, por sua ambição científica, impossibilitar ao paciente a condição da livre associação, arriscando conduzi-lo a produzir o resultado esperado sobre o tema pesquisado(18).

Este estudo foi aprovado pelo comitê de ética em pesquisa com seres humanos da Faculdade de Ciências Médicas da Unicamp, sob o parecer $n^{\circ}$ 59385616.6.0000.5404.

\section{Resultados}

\section{Discurso do sujeito: a relação enfermeiro-} paciente e a escuta dos significantes

O primeiro significante que surge no decorrer das primeiras consultas foi dado a partir do posicionamento do paciente (R.), sempre perpendicular a enfermeira, sem manter contato visual e raramente esboçando qualquer tipo de reação, independentemente do assunto tratado, que variava entre clima, futebol e convivência na residência terapêutica.

Nas primeiras quatro consultas foram realizadas medidas de glicemia capilar, cujos valores variaram entre $305 \mathrm{mg} / \mathrm{mL}$ e $365 \mathrm{mg} / \mathrm{mL}$. Tais valores foram sempre mostrados ao paciente, numa tentativa de observar qual seria sua reação, e a resposta sempre era está baixa, independentemente do número mostrado, embora R. tenha provado que conhecia os valores de normalidade da glicemia capilar.

O segundo significante foi adotado a partir do entendimento e efeito particular que a glicemia capilar possui para este sujeito que, a despeito do valor indicado e lido, sempre reconhece que a glicemia está baixa, expondo um fato paradoxal. Tal consideração, de uma glicemia alta tomada como baixa, ocorre quando o paciente se posiciona perpendicularmente a enfermeira.

Nas consultas, enquanto o paciente permanece em posição perpendicular, o aparecimento do terceiro significante é favorecido, no qual ele denomina as questões de seu adoecimento e tratamento a partir de um eles demandantes, pronome que usa para nomear a equipe que cuida dele com medicação e indicações de regime alimentar, os companheiros da moradia que sempre lhe pediam favores e até a própria enfermeira, para quem pedia permissão para tudo que fosse fazer. Assim, pergunta-se: ele mantém-se perpendicular ao próprio tratamento?

A leitura dos três primeiros significantes (posição perpendicular, seguida pelo paradoxo alto/ baixo da glicemia, que levou ao aparecimento do eles demandantes) possibilitou uma mudança no posicionamento diante da relação, chamada de momento de virada discursiva. Para tanto, a primeira intervenção de enfermagem adotada pela enfermeira foi a de colocar-se no lugar de alguém que não estava ali para pressioná-lo e que não priorizava seu peso e glicemia (elementos muito importantes para os demais membros da equipe).

Para conseguir realizar tal mudança no posicionamento, foi realizada uma consulta com o paciente, em um local onde houvesse oferta de alimentos.

Uma vez realizada essa intervenção, algo se alterou nas consultas subsequentes. O paciente começou a perguntar sobre a enfermeira para os demais membros da equipe, considerando-a como não havia feito antes, mudando a forma de se sentar, ficando de frente e estabelecendo contato visual. Além disso, passou a trazer assuntos que o incomodavam, como a pressão exercida pela equipe do Caps em relação à nova terapia medicamentosa, e temas em que se colocava como foco. Nas últimas consultas, R. falou mais fluidamente e deixou transparecer sentimentos de raiva e frustração, como solilóquios não presenciados até então e movimentos corporais imotivados. Enfim, R. pôde aparecer como sujeito para além do eles demandantes.

Como efeito, ele se mostrou também interessado em objetos (máquina fotográfica adquirida em um bazar e mesa encontrada na rua) e os obteve para si. Esses objetos eram sempre de grande interesse para o paciente, que fazia questão de mostrar e detalhar seu funcionamento e como poderia restaurá-los, além de, efetivamente, consertá-los. Porém, nas consultas subsequentes às que o paciente obtinha os objetos, ele sempre os havia trocado como forma de pagamento de dívidas.

\section{Discurso da família}

R. nunca falou de sua família, ela está presente apenas nas informações do prontuário, que traz histórico de abandono familiar seguido de seis anos morando em abrigo, relatos de mãe adotiva superprotetora e irmãos com os quais não possui nenhum contato. 
Mesmo essas poucas informações obtidas pelo prontuário marcam um vazio e uma ausência no lugar da família deste paciente.

\section{Discurso da instituição}

Os trabalhadores da instituição, que são os eles demandantes, têm como discurso que se trata de um paciente que não tem jeito, come demais, que não obedece a equipe e não segue o tratamento. A equipe repetiu essas informações para a enfermeira e para o próprio $\mathrm{R}$. durante o período em que ocorreram as consultas de enfermagem. Tal fato evidencia a valorização de determinados dados clínicos pela equipe, que são: o diagnóstico de retardo mental leve de base, caracterizado pela dificuldade escolar, histórico de internações psiquiátricas prévias devido à condição social e o comportamento agressivo. Atualmente a equipe enfatiza os diagnósticos de obesidade mórbida, hipercolesterolemia, hipertensão arterial sistêmica (HAS), diabetes mellitus (DM) tipo II e histórico de não adesão ao tratamento e à reeducação alimentar, associados ao contexto em que R. está, morador da residência terapêutica vinculada ao Caps.

Possui diagnóstico de esquizofrenia, apresentando alterações recorrentes ao exame de estado mental, observadas pela enfermeira e pela equipe: embotamento afetivo, hipoprosexia, avolição, hipomodulação do humor e afeto congruente, juízo crítico da realidade diminuído. Além disso, apresentou uma única vez polilalia, solilóquios e alteração psicomotora com movimentos repetitivos, involuntários e imotivados de membros superiores $^{(19)}$.

Em uso de medicamentos das classes: antidepressivo inibidor seletivo da receptação de serotonina, diurético tiazídico, classe das estatinas, anti-inflamatório não esteroide, inibidor da enzima conversora de angiotensina ( $\mathrm{iECA}$ ), hipoglicemiante oral e antidiabético.

\section{Discussão: proposta de cuidado e sua contribuição para o PTS}

Para iniciar a CCC-SM é importante ressaltar que a relação estabelecida entre enfermeiro e paciente fez emergir os significantes já apontados, porém outro profissional poderia tomar uma condução diversa, uma vez que a escuta pautada na relação conta com a subjetividade dos dois sujeitos em cena ${ }^{(14,16)}$.

Destaca-se que para a proposição do cuidado de enfermagem na perspectiva do sujeito do inconsciente é importante delimitar a função diagnóstica, sintomal e transferencial da relação de cuidado estabelecida ${ }^{(10)}$.

Para avançar nessa discussão, propõe-se olhar primeiramente para a questão do diagnóstico estrutural, a psicose, diagnosticada a partir da leitura da função sintomal da dissociação de R. entre seu corpo e suas escolhas e caracterizada pelo paradoxo entre os resultados de glicemia capilar e o rebaixamento crítico de sua percepção em relação a esses valores, favorecendo sua escolha por alimentos hipercalóricos. Utilizou-se também a leitura da posição perpendicular, que pode configurar o embotamento afetivo e sua condição de objeto de gozo diante do eles demandantes, como se os profissionais, ao zelarem pela sua saúde, permanecessem na posição de um Outro invasor, característico da psicose ${ }^{(20)}$.

Na psicose há a foraclusão do Nome-do-Pai, ou seja, o sujeito não passa pelo segundo tempo edipiano, no qual é apresentado à lei, à falta. Uma vez que a articulação da metáfora do Nome-do-Pai inaugura a simbolização, a foraclusão do Nome-do-Pai na psicose corresponde à não inserção na lei simbólica, fazendo com que o sujeito não seja submetido à castração simbólica(20).

Nesse sentido, o Outro do psicótico não inscreve a lei e a falta, sendo então um Outro absoluto e demandante, que reproduz o primeiro tempo lógico de Édipo e mantém o indivíduo na fase de espelho com a função mãe (a'), na qual o sujeito associa sua existência à de outrem ${ }^{(20)}$. Observa-se o movimento da estruturação psicótica determinada pela foraclusão da metáfora do Nome-do-Pai sempre que o paciente localiza os profissionais como eles demandantes, que determinam de forma imperiosa as ações de R.

Nesse caso, o esvaziamento do lugar da família colaborou com a estruturação psíquica de R., na medida em que as relações afetivas do começo da vida de um sujeito são a porta de entrada para a inscrição da metáfora do Nome-do-Pai. Assim, a relação especular e de rivalidade entre $\mathrm{R}$. e o eles demandantes permanece sob a condição de sua existência remetida a um Outro, que dele tudo sabe e tudo pode mandar ${ }^{(20)}$. O posicionamento perpendicular do paciente pode ser lido, neste caso, como uma defesa, que só pôde ser superada quando a enfermeira estabeleceu sua intervenção, caracterizada por colocar-se no lugar de alguém que não estava ali para pressioná-lo e que não priorizou o discurso da instituição, no caso o eles demandantes.

O psicótico mantém-se identificado à posição de gozo, oferecendo-se como objeto no lugar da falta que não se inscreveu pela castração(7). É pela via do objeto do gozo do Outro que o paciente significa no 
real (corpo) $)^{(20)}$, o que leva a uma dissociação entre o corpo e as escolhas do psicótico, constituindo assim o dito corpo despedaçado da psicose ${ }^{(21)}$.

No caso de R., o corpo despedaçado surge na dissociação entre os valores glicêmicos e seu julgamento quanto a eles, o que o mantém na opção por alimentos que não são apropriados a sua dieta, conduzindo a equipe a responder-lhe com ações invasivas de proibição do alimento, reforçando sua condição de objeto de gozo do outro. Quando há tal invasão, os profissionais ocupam a posição de grande Outro, impedindo que R. construa a condição de sujeito da própria vida, o que corrobora com o histórico de não adesão a tratamentos.

Ainda em relação à função diagnóstica, temos que o paciente débil, no contexto da clínica psicanalítica, funciona de modo a responder às demandas alheias, não assume o próprio desejo e toma como seu o desejo alheio, fazendo aquilo que o Outro Ihe demanda. Dessa forma, a clínica com a debilidade revela sempre uma impossibilidade de o sujeito de acessar seu saber inconsciente e seu próprio desejo, causando uma grande dificuldade de diagnóstico e manejo clínico ${ }^{(22)}$. Pode-se considerar, assim, que a debilidade mental e o déficit cognitivo são fatores que contribuíram para a dificuldade do paciente em se colocar em trabalho nas consultas, e tornaram-se elementos adicionais à psicose, mantendo-o na posição de submissão e resistente ao tratamento.

Há também a leitura da função sintomal, que pode ser definida como a demanda do paciente endereçada ao enfermeiro (na perspectiva da clínica do sujeito), e que é colocada como questão para o sujeito, para que seja instigado a decifrá-la na clínica do inconsciente ${ }^{(10)}$. No caso de R., evidenciou-se uma alternância entre o posicionamento perpendicular e não perpendicular frente à enfermeira, movimento este que caracterizou a via pela qual o sintoma de embotamento afetivo surgiu.

Nesse contexto, pode-se pensar na relação transferencial, na qual o paciente oferece à enfermeira o lugar do Outro absoluto ${ }^{(20)}$, por meio do Sujeito Suposto Saber ${ }^{(10)}$, identificado pela postura inicial adotada pela enfermeira em consonância com a equipe, ao focar-se principalmente nas questões fisiológicas, fazendo surgir o eles demandante e invasor.

Assim, articulando a função diagnóstica, sintomática e transferencial, a enfermeira localizou a transferência a partir do Sujeito Suposto Saber ${ }^{(10)}$ nas recomendações de cuidado realizadas para responder à condição de hipertensão e diabetes. No entanto, a organização da clínica da psicose demanda a construção da metáfora delirante, que faz suplência à falta da metáfora do Nomedo-Pai, barrando o gozo do outro ${ }^{(23)}$. Assim, no caso de R., a intervenção de enfermagem constitui-se pela virada da relação, deslocando a enfermeira do lugar de Sujeito Suposto Saber, configurando um "fazer diferente" que, neste caso, delimitou-se por fazer o inverso do esperado, convidando-o a comer. A partir desse convite, $R$. se posicionou de frente para a enfermeira e trouxe para a cena do cuidado a sua escolha por objetos, retirando-se da condição de objeto, constituindo-se, assim, a metáfora delirante pelo restauro dos objetos encontrados na rua e que são relatados durante as consultas de enfermagem.

Reforça-se aqui que não se considera incorreto o manejo da equipe. Considera-se apenas que era necessário, além do que já era feito, haver alguém para receber o sintoma da forma que ele se constituísse e servisse de intermédio para o paciente trabalhar seus significantes e barrar o gozo do Outro, fazendo surgir um distanciamento entre ele e o Outro, instaurando o paciente como sujeito(20)

Considera-se, enfim, que as formas de cuidado precisam ser repensadas a cada dia, para cada sujeito, não havendo um modelo a ser seguido, repetido ou passível de padronização(2). Propõe-se, assim, desenvolver um cuidado que considere o sujeito em sua singularidade, o qual deve ser acolhido e pensado na sua diferença $a^{(6)}$, incorporando estratégias e modalidades terapêuticas favorecedoras da retomada do paciente ao seu lugar de sujeito(2).

Porém, em meio a tantas possibilidades, há uma ferramenta essencial que deve ser sempre utilizada: a escuta, uma vez que se considera que a abordagem do sujeito somente será viável pela possibilidade de o paciente recorrer à própria fala ${ }^{(6)}$. É através da fala que o sujeito surgirá como efeito único de linguagem, que se apresenta sob a forma de uma cadeia de significantes construída à sua maneira ${ }^{(6,9)}$.

O essencial das consultas de enfermagem é, também, que o enfermeiro seja aquele que se ofereça como suporte para todas as demandas e que não responda a nenhuma ${ }^{(24)}$. Dessa forma, a estratégia aqui elucidada mostra uma opção de como introduzir uma relação terapêutica com o paciente, deslocando o enfermeiro da posição de Sujeito Suposto Saber, que no caso da psicose corrobora com a invasão, e convidando o paciente a ocupar uma posição diferente da de objeto de desejo do Outro ${ }^{(20)}$.

O projeto terapêutico, em si, tem como essência considerar a singularidade do indivíduo, discutida até agora, valorizando seu modo de compreender a vida, suas subjetividades e singularidades ${ }^{(25)}$, sendo determinante da ação de saúde oferecida, objetivando cuidar, melhorar a qualidade de vida dos usuários, 
ampliar o conhecimento e a apropriação do processo saúde-doença, entre outros ${ }^{(3)}$.

Lembrando que cada estratégia deve ser usada para aquele paciente, naquele momento, com base naquela relação desenvolvida. Não se pretende, com este trabalho, fornecer uma ideia de intervenção a ser usada em outros pacientes, mas fornecer subsídio para que ações, muitas vezes consideradas alternativas e incomuns, sejam justificadas ao considerar todo o contexto daquele paciente e a relação estabelecida com aquele que the presta cuidado, podendo-se utilizar a proposta do PTS como um instrumento de trabalho para o caso a caso e não como um protocolo a ser cumprido(25).

A estratégia de mudança de posição na relação terapêutica com o paciente, descrita neste trabalho, se pensada fora de contexto e sem conhecimento do desenrolar das consultas, poderia ser vista como inadequada, por exemplo, quando o paciente é convidado a se alimentar, a despeito de todas as restrições clínicas que possui. Entretanto, ao considerar o PTS proposto, percebe-se que uma ação que para outro paciente não teria qualquer significado, para $\mathrm{R}$. surtiu bons efeitos.

Por fim, destaca-se também que o PTS assume a característica de fazer a equipe refletir sobre sua própria prática, seja ela individual ou coletiva. Ele possibilita o reconhecimento de fragilidades, potencialidades, necessidades e demandas, favorecendo uma mudança necessária no cuidado psicossocial, por meio de alternativas e ações baseadas em novas tecnologias em saúde, como as relacionais, que considerem a singularidade e a corresponsabilização(3).

\section{Considerações Finais}

A construção da proposta de cuidado de enfermagem a partir da leitura do sujeito do inconsciente, por meio do método da Construção de Caso Clínico, foi organizada a partir do cotejamento do discurso do sujeito, da família e da instituição, o que favoreceu a identificação das funções diagnóstica, sintomal e transferencial. Tal percurso teórico caracterizou-se como uma contribuição para a enfermagem, pois possibilitou constituir um cuidado pautado pelo "fazer diferente", que muitas vezes pode parecer contraditório à prática desenvolvida no cotidiano. Assim, obteve-se uma resposta inovadora, a possibilidade de deslocamento do sujeito para uma posição em que se destaca sua responsabilidade sobre seu viver e sofrer. Tal construção é a colaboração que o enfermeiro, a partir da perspectiva da clínica do sujeito, traz para a elaboração do PTS.

\section{Referências}

1. Garcia APRF, Freitas MIP, Lamas JLT, Toledo VP. Processo de enfermagem em saúde mental: revisão integrativa da literatura. Rev Bras Enferm. 2017;70(1):209-18.

2. Kurimoto, TCS. O cuidado de enfermagem em saúde mental na perspectiva da clínica do sujeito: questões de fineza. Rev Bras Enferm. No prelo 2018.

3. Vasconcelos MGF, Jorge MSB, Catrib AMF, Bezerra IC, Franco TB. Therapeutic design in Mental Health: practices and procedures in dimensions constituents of psychosocial care. Interface Comun Saúde Educ. 2016; 20(57):313-23.

4. Boccardo ACS, Zane FC, Rodrigues, S, Mângia EF. O projeto terapêutico singular como estratégia de organização do cuidado nos serviços de saúde mental. Rev Ter Ocup. 2011;22(1):85-92.

5. Bliss S, Baltzly D, Bull R, Dalton L, Jones J. A role for virtude in unifying the "knowledge" and "caring" discourses in nursing theory. Nurs Inq. 2017;00:e12191.

6. Vieira AN, Silveira LC, Silva LMS, Rodrigues DP, Martins IC. Reflexão acerca da contribuição da psicanálise para o cuidado e para a clínica de enfermagem. Rev Enferm UFPE on line. 2014; 8(2):450-6.

7. Lacan J. O Seminário livro 4: a relação de objeto. Rio de Janeiro (RJ): Jorge Zahar Ed; 1995. 380 p.

8. Lacan J. Função e campo da fala e da linguagem em psicanálise. In: Lacan J. Escritos. 2 ed. Rio de Janeiro (RJ): Jorge Zahar Editor; 1998. p. 944.

9. Pergola AM, Gargia APRF. O aprendizado na construção de caso clínico em saúde mental. Rev Esc Enferm USP. 2008; 42(2):383-8.

10. Quinet A. As 4+1 condições da análise. 12 ed. Rio de Janeiro (RJ): Jorge Zahar Ed; 2009. 115 p.

11. Viganò $C$. A construção do caso clínico em saúde mental. Rev Curinga. 1999; 1(13):39-48.

12. Silveira LC, Paiva Filho F. Psicose e drogadição: a construção do caso na clínica de enfermagem. SMAD Rev Eletrônica Saúde Mental Álcool Drog. 2014; 10(1):29-34. 13. Ravanello T, Machado ID, Martinez MC, Nabarrete LMS. Os caminhos da pesquisa psicanalítica: da epistemologia ao método. Gerais. 2016; 9(1):110-24.

14. Iribarry IN. O que é pesquisa psicanalítica? Agora. 2003; 6(1):115-38.

15. Longo L. Linguagem e psicanálise. Rio de Janeiro (RJ): Jorge Zahar Ed; 2006. 72 p.

16. Viganò $C$. A construção do Caso Clinico. Opção Lacaniana. 2010; 1(1):1-9.

17. Freud S. A dinâmica da transferência. In: Freud S. O caso Schereber: artigos sobre técnica e outros trabalhos. São Paulo (SP): Companhia das Letras; 2010. 392. p.

18. Freud S. Recomendações aos médicos que exercem a psicanálise. In: Freud S. O caso Schereber: artigos sobre 
técnica e outros trabalhos. São Paulo (SP): Companhia das Letras; 2010. 392. p.

19. Dalgalorrondo P. Síndromes psicóticas: quadros do espectro da esquizofrenia e outras psicoses. In: Dalgalorrondo P. Psicopatologia e semiologia dos transtornos mentais. 2 ed. Porto Alegre (RS): Artmed; 2008. 439. p.

20. Quinet A. Teoria e Clínica da Psicose. 3 ed. Rio de Janeiro (RJ): Forense Universitária; 2006. 238 p.

21. Miller J-A. Lacan elucidado: palestras no Brasil. Rio de Janeiro (RJ): Jorge Zahar Ed; 1997. 611 p.

22. Sanches DR, Berlinck MT. Debilidade mental: o patinho feio da clínica psicanalítica. Agora. 2010; 13(2):259-74.

23. Guerra AMC. A estabilização psicótica na perspectiva borromeana: criação e suplência [Tese]. Rio de Janeiro (RJ): Universidade Federal do Rio de Janeiro; 2017. 270 p. 24. Lacan J. O seminário livro 5: As formações do inconsciente. Rio de Janeiro (RJ): Jorge Zahar Editor; 1999. $364 \mathrm{p}$.

25. De Luccia D, Freitas CCS, Di Ribeiro DL, Saccani LP, Braga MB, Valle SHA, et al. O Protagonismo no Projeto Terapêutico Singular: Singularidade e implicação do sujeito no nível terciário em saúde. A peste: Revista de Psicanálise e Sociedade e Filosofia. 2016; 7(1):49-64. 